\title{
Sensitivity Study of an Ultrasound coupled Transrectal Electrical Impedance Tomography System for Prostate Imaging
}

\author{
Y. Wan ${ }^{1}$, R. Halter ${ }^{1}$, A. Borsic ${ }^{1}$, P. Manwaring ${ }^{1}$, A. Hartov ${ }^{1}$, and K. Paulsen ${ }^{1}$ \\ ${ }^{1}$ Thayer School of Engineering, Dartmouth College, NH 03755, USA
}

\begin{abstract}
In 2009, prostate cancer ranks as the most common cancer and the second most fatal cancer in men in the United States. Unfortunately, the current clinical diagnostic methods (e.g. prostatespecific antigen (PSA), digital rectal examination, endorectal MRI, transrectal ultrasound, biopsy) used for detecting and staging prostate cancer are limited. It has been shown that cancerous prostate tissue has significantly different electrical properties when compared to benign tissues. Based on these electrical property findings, a TransRectal Electrical Impedance Tomography (TREIT) system is proposed as a novel prostate imaging modality. The TREIT system is comprised of an array of electrodes interfaced with a clinical TransRectal UltraSound (TRUS) probe. We evaluate this imaging system through series of phantom imaging experiments to assess the system's ability to image high and low contrast objects at various positions. We found that the TREIT system can easily discern high contrast inclusions of $1 \mathrm{~cm}$ in diameter at distances centered at 2 times the radius of the TREIT probe away from the probe surface. Furthermore, this technology's ability to detect low contrast inclusions suggests that it has the potential to successfully detect prostate cancer.
\end{abstract}

\section{Keywords}

Electrical Impedance Tomography; Ultrasound; prostate cancer; sensitivity; reconstruction

\section{Introduction}

In 2009, prostate cancer ranks as the most common cancer and the second most fatal cancer in men in the United States. Approximately 192,280 men in the United States are expected to be diagnosed with prostate cancer in 2009, and 27,360 men are expected to die from it [1]. Currently, clinical diagnostic methods including prostate-specific antigen (PSA) level, digital rectal examination, endorectal MRI, Transrectal Ultrasound (TRUS), biopsy, etc., are limited to detect prostate cancer.

Halter et al reported that cancerous tissue has significant different conductivity and permittivity than that of glandular or stroma tissue in the prostate at frequencies ranging from $0.1 \mathrm{kHz}$ to $100 \mathrm{kHz}$ [3]. Based on these findings, we developed a TransRectal Electrical Impedance Tomography (TREIT) modality to distinguish cancerous tissue from surrounding tissue in the prostate by sensing these significant differences in electrical properties. 
Borsic et al performed a three dimensional simulation-based analysis of a TREIT probe [4] and showed that there was sufficient sensitivity to suggest the feasibility of using this system. In this study, we describe our imaging hardware and analyze the sensitivity of it when combined with the reconstruction algorithm presented by Borsic et al [2].

\section{Methods}

The TREIT system is incorporated into a standard TRUS probe (TargetScan from Envisioneering Medical Technologies, St. Louis, MO, USA) to provide a multi-modal imaging device. The ultrasound (US) provides anatomic information of the prostate and peri-prostatic space, which can be used as a priori information to generate finite element method (FEM) meshes of the prostate gland and guide Electrical Impedance Tomography (EIT) image reconstruction. A flexible kapton circuit with thirty electrodes lying along the periphery of the acoustic window without impeding the ultrasound field (shown in Figure 1(a)) bridges the electrodes and the data acquisition (DAQ) system, which is comprised of a custom built front-end switching network interfaced to a NI USB 6259 data acquisition unit (National Instruments, Austin, Texas). The data acquisition scheme involves driving an AC voltage at $3.2 \mathrm{kHz}$ through a pair of electrodes and sensing passive voltages on the remaining 28 electrodes. An average channel SNR is $81 \mathrm{~dB}$.

A suite of phantom experiments were employed to assess the imaging capabilities of this system. The TREIT probe is submerged into a tank filled with a saline solution so that the probe tip points downward. The conductivity of the saline solution was measured with a conductivity meter (CDH222) to be $0.1 \mathrm{~S} / \mathrm{m}$. The radius of the tank is $12 \mathrm{~cm}$, and the height of the saline bath is $20 \mathrm{~cm}$. Inclusions are submerged into the saline bath, and positioned in front of the the electrodes for imaging (Figure 1(b)). In order to better describe the position of the inclusion, it is convenient to view the imaging system in a cylindrical coordinate system. Within this system, the inclusion center is defined by its distance from the outer surface of the probe $(\rho)$, the angle spanned between it and the center of the acoustic window $(\phi)$, and its height relative to the lower edge of the acoustic window $(z)$.

For this study, three specific factors a ecting the sensitivity of the TREIT system are analyzed:

(i) Size

Spherical stainless steel balls of four different sizes are used as high contrast inclusions to assess image sensitivity to inclusion size. The radii of the balls are $R_{1}=1.03 \mathrm{~cm}, R_{2}=0.76 \mathrm{~cm}, R_{3}=0.64 \mathrm{~cm}, R_{4}=0.52 \mathrm{~cm}$.

(ii) Position

A spherical stainless steel ball with radius $R_{3}=0.64 \mathrm{~cm}$ is imaged at different positions to assess regional sensitivity. The dimensional sensitivity assessed includes:

(a) Radial distance from the probe: the inclusion is placed at $\rho_{1}=0.69$ $\mathrm{cm}, \rho_{2}=1.51 \mathrm{~cm}, \rho_{3}=2.30 \mathrm{~cm}, \rho_{4}=3.93 \mathrm{~cm}$, and $\rho_{5}=5.00 \mathrm{~cm}$ with $\phi=0^{\circ}$ and $z=3 \mathrm{~cm}$ fixed.

(b) Angular position: the inclusion is placed at $\phi_{1}=26.85^{\circ}, \phi_{2}=0^{\circ}$, and $\phi_{3}=-27.58^{\circ}$ with $\rho=1.71 \mathrm{~cm}$ and $z=3 \mathrm{~cm}$ fixed.

(c) Height: the inclusion is positioned at $z_{1}=4.8 \mathrm{~cm}, z_{2}=3 \mathrm{~cm}$, and $z_{3}$ $=1.2 \mathrm{~cm}$ with $\rho=1.71 \mathrm{~cm}$ and $\phi=0^{\circ}$ fixed.

(iii) Conductivity 
Inclusions of different conductivity are created by filling an electrically conductive enclosure (natural collagen-based membrane) [5] with saline solutions of various $\mathrm{NaCl}$ concentrations to assess sensitivity to inclusion contrast. Eight saline solutions with conductivities ranging from $0.02 \mathrm{~S} / \mathrm{m}$ to $1 \mathrm{~S} /$ $\mathrm{m}$ are tested and compared in this study.

\section{Results}

The TREIT reconstructed images are shown in Figure 2. Conductivity is plotted along a horizontal line through the center of the imaged inclusion (Figure 3). The reconstructed inclusion radius decreases linearly with the size of the actual inclusion, indicating that the TREIT system is able to accurately distinguish between different sized high contrast objects. For distant inclusions $\left(\rho_{4}\right.$ and $\left.\rho_{5}\right)$, there are no well defined regions of high conductivity observed near where the inclusion is positioned. For nearfield $\left(\rho_{1}\right.$ and $\left.\rho_{2}\right)$, the reconstructed inclusions more accurately depict the correct inclusion position. The reconstructions of inclusions at different angular positions and height showed that the TREIT probe has slightly better sensitivity in the middle than at the edge of the electrode array. In addition, the reconstructed inclusions at the edge of the acoustic window appeared closer to the middle than their actual position. In the reconstructed images of different conductivity, it is obvious that the reconstructed conductivity gradually increases with the true inclusion conductivity. For inclusion conductivities of $0.1 \mathrm{~S} / \mathrm{m}$ and $0.12 \mathrm{~S} / \mathrm{m}$, it is very difficult to discern the inclusion from the background. For higher conductivity contrasts, it is relatively easy to distinguish inclusions from the background in the reconstructed image.

\section{Discussion}

For high contrast inclusions of different sizes, the reconstructed object is clearly dependent on the inclusion radius. The ratio between reconstructed inclusion radius and true inclusion radius ranges from 2 to 2.4 . High contrast inclusions of $1 \mathrm{~cm}$ diameter are easily observed and we expect that smaller high-contrast inclusions will also be detectable. Similarly sized inclusions have been reconstructed with closed-domain EIT systems [6][7].

The TREIT probe loses sensitivity at $\rho_{4}=3.93 \mathrm{~cm}$ and is regarded as an estimate of the limit in radial sensitivity. The conductivity and size of the reconstructed inclusions are somewhat dependent on the distance from the probe surface. Smaller current densities exist far from the probe surface as compared to the probes near field, leading to a decreased far-field sensitivity. Jossinet et al [8] found that the radial sensitivity for an open-domain EIT imaging was limited to detecting objects centered at a distance of 2 to 3 radii from the surface of the probe. We found that at a radial distance of $2.30 \mathrm{~cm}$ the inclusion was accurately gauged. This distance represents 2 times the radius of the probe (radius $=1.14$ $\mathrm{cm})$. The inclusion appears to be closer to the probe in reconstructed image for $\rho_{3}$ than $\rho_{2}$, however the conductivity value of the reconstructed inclusion at $\rho_{3}$ is much smaller than that at $\rho_{2}$. This positional inaccuracy $\rho_{3}$ results from a non-unique solution to this ill-conditioned EIT inverse problem. The conductivity distribution in the reconstructed image of $\rho_{3}$ produces voltages at the electrodes matching the experimental measurements. Alternatively stated, the positional inaccuracies are compensated for by the decrease in reconstructed inclusion conductivity. Having ultrasound as a guidance with a priori geometric information is expected to better condition the problem and improve the accuracy of the reconstructed image.

For the angular positions, the maximum accuracy occurs at $0^{\circ}$, which is in the middle of the electrode window. Similarly, as the z-location of the inclusion was adjusted the maximum accuracy was observed when it was centered within the acoustic window. This occurs 
because the current density is larger flowing through the central regions. Despite different arrangement of the electrode array, Borsic et al [4] showed similar results in their theoretical sensitivity analysis. Specifically, the anterior and lateral aspects of the imaging domain will have the lowest sensitivity, while the central pixels closest to the probe surface have optimal sensitivity.

In the low contrast sensitivity analysis, we are able to assess conductivity variation $2 \mathrm{~cm}$ away from probe face as evidenced by the well defined spherical reconstruction. The smallest discernible inclusion conductivity is $0.08 \mathrm{~S} / \mathrm{m}$ in a saline background solution of 0.1 $\mathrm{S} / \mathrm{m}$, and the contrast is $(1.0-0.08) / 0.1=20 \%$. This level of contrast is similar to that observed in conductivity between cancerous and stroma tissues (23\%) at $10 \mathrm{kHz}$ and is much less than the contrasts observed in permittivity between benign and malignant tissues (43-63\%), reported by Halter et al [3]. As a result, we expect this TREIT system to be able to distinguish cancerous from benign prostate tissue.

\section{Conclusion}

In conclusion, we demonstrated that our TREIT imaging system is able to image high contrast inclusions of at least $1 \mathrm{~cm}$ in diameter, and the effective range is 2 times the radius of the probe. The TREIT probe can easily distinguish high contrast inclusions located at different angular positions as well as different heights. In addition, the imaging system is capable of detecting inclusions of $20 \%$ conductivity contrast to the background. Currently, we are collecting clinical data with the system. Future work includes co-registeration of TREIT reconstructions with US images and incorporation of the anatomic information gleaned from US images into the TREIT reconstruction algorithm.

\section{Acknowledgments}

This work is supported by the NIH grants 5R01CA124925 and 1RC1EB011000-01.

\section{References}

[1]. Jemal A, Siegal R, Ward E, Hao Y, Xu J, Thun M. Cancer Statistics, 2009. CA Cancer J. Clin 2009. 2009; 59

[2]. Borsic A, Halter R, Wan Y, Hartov A, Paulsen KD. Electrical Impedance Tomography Reconstruction for Three Dimensional Imaging of the Prostate. Physiological Measurement. 2009

[3]. Halter RJ, Schned A, Heaney J, et al. Electrical Properties of Prostatic Tissues: I. Single Frequency Admittivity Properties. Journal of Urology 2009-10182. 2009; 4

[4]. Borsic A, Halter R, Wan Y, Hartov A, Paulsen KD. Sensitivity study and optimization of a 3D electric impedance tomography prostate probe. Physiological Measurement 2009 0630. 2009; 6

[5]. Manwearing PK, Wan Y, Halter R, Hartov A. A natural membrane material enabling multi-layer saline tank phantoms for use in EIT. Physiological Measurement. 2009

[6]. Halter, Ryan J.; Hartov, Alex; Paulsen, Keith D. A Broadband High-Frequency Electrical Impedance Tomography System for Breast Imaging. IEEE Transactions on Biomedical Engineering. Feburary.2008 55(2) 2008.

[7]. Saulnier; Liu, GJN.; Tamma, C.; Xia, H.; Kao, T-J.; Newell, JC.; Isaacson, D. An Electrical Impedance Spectroscopy System for Breast Cancer Detection. Annual International Conference of the IEEE Engineering in Medicine and Biology; 2007. 2007

[8]. Jossinet, Jacques; Marry, Emmanuel; Matias, Adrien. Electrical Impedance Endotomography. Phys. Med. Biol. 472002 21892202. 2002 


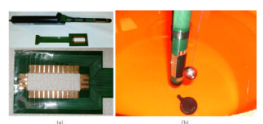

Figure 1.

(a) Upper: flex circuit of electrodes wrapped around the ultrasound probe; lower: magnified view of flex circuit of electrodes laid flat. (b) Imaging configuration: the inclusion is placed immediately in front of the acoustic window.. 


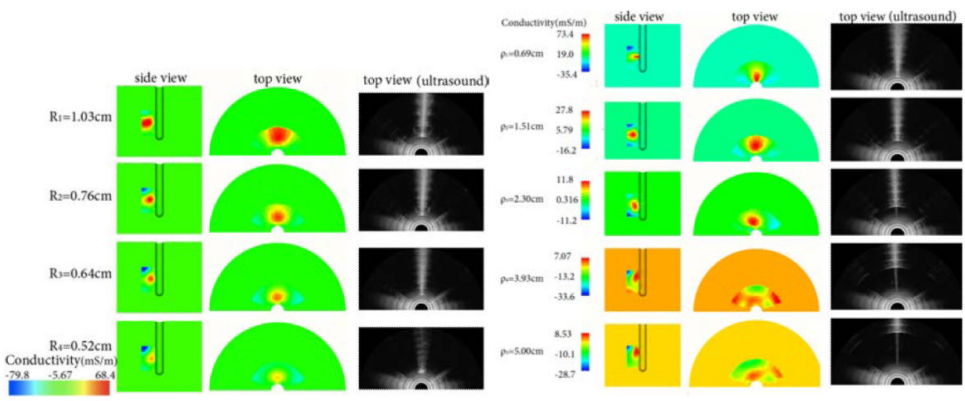

(a)

(b)

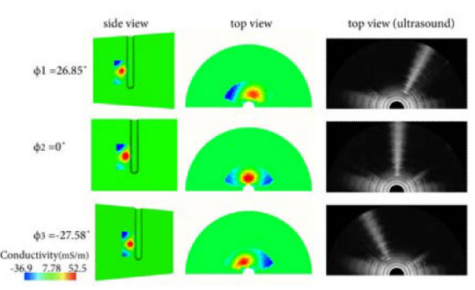

(c)

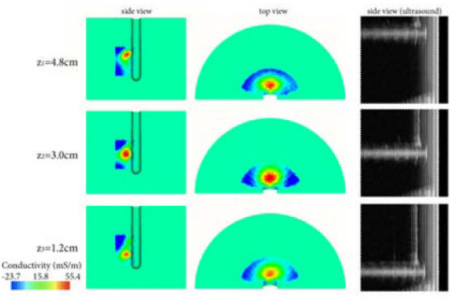

(d)

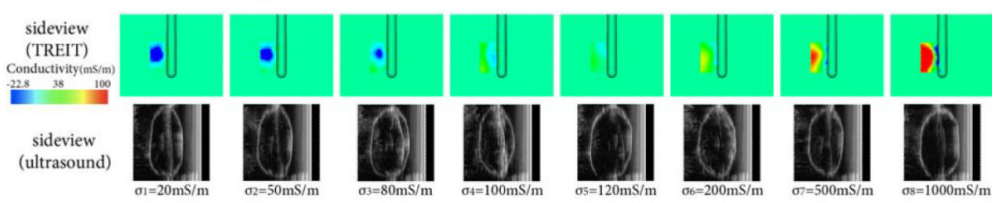

(e)

Figure 2.

TREIT reconstructed images: (a) with high contrast inclusions of 4 different sizes; (b) with high contrast inclusions at 5 different radial distances from the probe; (c) with high contrast inclusions at 3 different angular positions; (d) with high contrast inclusions at 3 different heights; (e) with inclusions of different contrasts. Transverse ultrasound images are displayed as a reference of inclusion sizes in (a) and inclusion positions in (b) and (c), and side views of ultrasound images are shown as a reflection of the inclusions at different heights in (d) and the shapes and positions of low contrast inclusions in (e). In the ultrasound images in (a), (b), (c) and (d), artifacts (bright tails after inclusions) are induced by mismatch of ultrasound reflection index of stainless steel ball and the saline solution. 


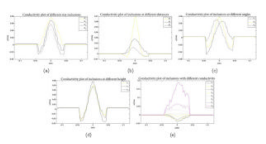

Figure 3.

Conductivity plot over a line going through the center of the reconstructed inclusion: (a) with high contrast inclusions of 4 different sizes; (b) with high contrast inclusions at 5 different radial distances from the probe; (c) with high contrast inclusions at 3 different angular positions; (d) with high contrast inclusions at 3 different heights; (e) with inclusions of different contrasts. 